\title{
Numerical Solution of Second-Order Linear Fredholm Integro-Differetial Equations by Trigonometric Scaling Functions
}

\author{
Hamid Safdari, Yones Esmaeelzade Aghdam \\ Department of Mathematics, Shahid Rajaee Teacher Training University, Tehran, Iran \\ Email: $\underline{\text { HSafdari@srttu.edu, yonesesmaeelzade@gmail.com }}$
}

Received 17 March 2015; accepted 13 April 2015; published 22 April 2015

Copyright (C) 2015 by authors and Scientific Research Publishing Inc.

This work is licensed under the Creative Commons Attribution International License (CC BY). http://creativecommons.org/licenses/by/4.0/

cc) (i) Open Access

\section{Abstract}

The main aim of this paper is to apply the Hermite trigonometric scaling function on [0, $2 \pi]$ which is constructed for Hermite interpolation for the linear Fredholm integro-differential equation of second order. This equation is usually difficult to solve analytically. Our approach consists of reducing the problem to a set of algebraic linear equations by expanding the approximate solution. Some numerical example is included to demonstrate the validity and applicability of the presented technique, the method produces very accurate results, and a comparison is made with exiting results. An estimation of error bound for this method is presented.

\section{Keywords}

Numerical Technique, Fredholm Integro-Differential Equations, Hermite Trigonometric Wavelets, Operational Matrix, Error Estimates

\section{Introduction}

In this paper we solve the Fredholm Linear Integro-Differential Equations as

$$
\mu_{0}(x)+\mu_{1}(x) u^{\prime}(x)+\mu_{2}(x) u^{\prime \prime}(x)=g(x)+\int_{0}^{1} k(x, t) u(t) \mathrm{d} t, \quad 0 \leq x \leq 1, u(0)=u_{0}, u(1)=u_{1}
$$

where $\mu_{i}(x), f(x)$, and $K_{l}(x, t)$ are given functions that have suitable derivatives, and $u_{0}$ and $u_{1}$ are given real constans. In most situations, it is difficult to obtain exact solution of the above integration. Hence various approximation method have been proposed and studied. The purpose of the present paper is to develop a trigonometric Hermite wavelet approximation for the computing of the problem [1]. 
Systems of integro-differential equations have a major role in the fields of science, physical phenomena, and engineering, such as nano-hydrodynamics, glass-forming process, dropwise condensation, wind ripple in the desert, and modeling the competition between tumor cells and the immune system. The concept of a system of integro-differential equations has motivated a huge amount of research work in recent years. Alot of attention has been devoted to the study of differential-difference equations, e.g. equations containing shifts of the unknown function and its derivates, and also integro-differential-difference equations. For instance, see [2] [3]. There are several numerical methods for solving system of linear integro-differential equations, for example, the rationalized Haar functions method [4], Galerkin methods with hybrid functions [5], the spline approximation method [6], the Chebyshev polynomial method [7], the spectral method [8], the CAS wavelet method [9], RugeKutta methods [10], the Adomian decomposition methods [11], and the interested reader can see [12] [13] for more published research works in the subject.

Our approach consists of reducing the problem to a set of linear equations by trigonometric scaling functions which is constructed for Hermite interpolation. A difficulty of using wavelet for the representation of integral operators is that quadrature leads to potentially high cost with sparse matrix. This fact particularly encourages us in efforts to devote to some appropriate wavelet bases to simplify the computation expense of the reoresentation matrix, which is importent to improve the wavelet method. Recently, the trigonometric interpolant wavelet has arisen in the approximation of operators [14]-[16]. Quack [17] has constructed a multiresolution analysis (MRA). Chen [18] [19] presented the feasibility of trigonometric wavelet numerical methods for stokes problem and Hadamard integral equation.

The organization of the rest of this paper is as follows: Section (0) describe the trigonometric scaling function on $[0,2 \pi]$, and construct the operational matrix of derivative for these function. Section $(0)$ summarizes the application of trigonometric scaling functions to the solution of Problem (1). Thus, a set of linear equations is formed and a solution of the considered problem is introduced. In Section (0), we report our computational results and demonstrate the accuracy of the proposed numerical schemes by presenting numerical examples. Note that we have computed the numerical results by MATLAB programming.

\section{Interpolatory Hermite Trigonometric Wavelets}

In this section, we will give a brief introduction of Quak's work on the construction of Hermite interpolatory trigonometric wavelets and their basic properties. More details can be found in (see [17]).

For all $j \in \mathbb{N}=\mathbb{N} \cup\{0\}$, two scaling functions $\varphi_{j, 0}^{0}(x)$ and $\varphi_{j, 0}^{1}(x)$ are defined as

$$
\begin{aligned}
\varphi_{j, 0}^{0}(x) & =\frac{1}{2^{2 j+1}} \sum_{l=0}^{2^{j+1}-1} D_{l}(x) \\
\varphi_{j, 0}^{1}(x) & =\frac{1}{2^{2 j+1}}\left(\tilde{D}_{2^{j+1}}(x)+\frac{1}{2} \sin \left(2^{j+1} x\right)\right)
\end{aligned}
$$

where the Dirichlet kernel $D_{l}(x)$ and its conjugate kernel $\tilde{D}_{l}(x)$ are defined as

$$
\begin{aligned}
& D_{l}(x)=\frac{1}{2}+\sum_{k=1}^{l} \cos k x= \begin{cases}\frac{\sin \left(l+\frac{1}{2}\right) x}{2 \sin \frac{x}{2}} & x \notin 2 \pi \mathbb{Z} \\
l+\frac{1}{2} & x \in 2 \pi \mathbb{Z}\end{cases} \\
& \tilde{D}_{l}(x)=\sum_{k=1}^{l} \sin k x= \begin{cases}\frac{\cos \frac{x}{2}-\cos \left(l+\frac{1}{2}\right)}{2 \sin \frac{x}{2}} & x \notin 2 \pi \mathbb{Z} \\
0 & x \in 2 \pi \mathbb{Z}\end{cases}
\end{aligned}
$$

Obviously, $D_{l}(x), \tilde{D}_{l}(x) \in T_{l}$, where $T_{l}$ is the linear space of trigonometric polynomials with degree not exceeding $l$. The equally spaced nodes on the interval $[0,2 \pi)$ with a dyadic step are denoted by $t_{j, l}=\frac{l \pi}{2^{j}}$, for 
any $j \in \mathbb{N}_{0}$ and $l=0,1, \cdots, 2^{j+1}-1$, where $\mathbb{N}_{0}$ is the set of all non-negative integers. Let $\varphi_{j, l}^{s}(x)=\varphi_{j, 0}^{s}\left(x-t_{j, l}\right)$, for $s=0,1$, and $l=0,1, \cdots, 2^{j+1}-1$.

Lemma 1 (See [17].) For $j \in \mathbb{N}_{0}$, we have

$$
\begin{aligned}
& \varphi_{j, 0}^{0}(x)= \begin{cases}\frac{1}{2^{2 j+2}} \frac{\sin ^{2}\left(2^{j} x\right)}{\sin ^{2}\left(\frac{x}{2}\right)} & x \notin 2 \pi \mathbb{Z} \\
1 & x \in 2 \pi \mathbb{Z}\end{cases} \\
& \varphi_{j, 0}^{1}(x)= \begin{cases}\frac{1}{2^{2 j+2}}\left(1-\cos \left(2^{j+1} x\right)\right) \cot \left(\frac{x}{2}\right) & x \notin 2 \pi \mathbb{Z} \\
0 & x \in 2 \pi \mathbb{Z}\end{cases}
\end{aligned}
$$

and their derivations are given by

$$
\begin{aligned}
& \left(\varphi_{j, 0}^{0}(x)\right)^{\prime}= \begin{cases}\frac{1}{2^{j+2}} \frac{\sin \left(2^{j+1} x\right)}{\sin ^{2}\left(\frac{x}{2}\right)}-\frac{1}{2^{2 j+2}} \frac{\sin ^{2}\left(2^{j} x\right) \cot \left(\frac{x}{2}\right)}{\sin \left(\frac{x}{2}\right)} & x \notin 2 \pi \mathbb{Z} \\
0 & x \in 2 \pi \mathbb{Z}\end{cases} \\
& \left(\varphi_{j, 0}^{1}(x)\right)^{\prime}= \begin{cases}\frac{1}{2^{2 j+3}} \frac{\cos \left(2^{j+1} x\right)-1}{\sin ^{2}\left(\frac{x}{2}\right)}+\frac{1}{2^{j+1}} \sin \left(2^{j+1} x\right) \cot \left(\frac{x}{2}\right) & x \notin 2 \pi \mathbb{Z} \\
1 & x \in 2 \pi \mathbb{Z}\end{cases}
\end{aligned}
$$

Theorem 1 (Interpolatory properties of the scaling functions). (See [17]) For $j \in \mathbb{N}_{0}$, the following interpolatory properties hold for each $l, k=0,1, \cdots, 2^{j+1}-1$

$$
\begin{array}{ll}
\varphi_{j, l}^{0}\left(x_{j, k}\right)=\delta_{k, l}, & \left(\varphi_{j, l}^{0}\left(x_{j, k}\right)\right)^{\prime}=0, \\
\varphi_{j, l}^{1}\left(x_{j, k}\right)=0, & \left(\varphi_{j, l}^{1}\left(x_{j, k}\right)\right)^{\prime}=\delta_{k, l} .
\end{array}
$$

From above we can take wavelet functions $\varphi_{j, l}^{0}(x), \varphi_{j, l}^{1}(x), l=0,1, \cdots, 2^{j+1}-1$ as scaling functions. Now, we can define the scaling function spaces $V_{j}$. Then we have

Definition 2 (Scaling functions space). For all $j \in \mathbb{N}_{0}$ define the wave space $V_{j}$ as follows

$$
V_{j}=\operatorname{span}\left\{\varphi_{j, n}^{0}(t), \varphi_{j, n}^{1}(t), n=0,1, \cdots, 2^{j+1}-1\right\}
$$

As a first step of studying the spaces $V_{j}$, the following result identifies the trigonometric polynomials which from alternative bases of these spaces.

Now a Hermite-type project operator can be introduced by means of the scaling functions. For all $j \in \mathbb{N}_{0}$ the Hermite projection operators $L_{j}$ mapping any real-valued differentiable $2 \pi$-periodic function $f$ into the space $V_{j}$ is defined as

$$
f(x) L_{j} f(x) \simeq \sum_{k=0}^{2^{j+1}-1}\left[a_{k} \varphi_{j, k}^{0}(x)+b_{k} \varphi_{j, k}^{1}(x)\right]=C^{\mathrm{T}} \Phi
$$

where $a_{k}=f\left(t_{j, k}\right), b_{k}=f^{\prime}\left(t_{j, k}\right), C$, and $\Phi$ are vectors with dimension $2^{j+2} \times 1$. The following properties of the operators $L_{j}$ are therefore obvious:

$$
\begin{gathered}
L_{j} \in T_{2^{j+1}} \\
L_{j} f\left(x_{j, k}\right)=f\left(x_{j, k}\right) \text { and }\left(L_{j} f\right)^{\prime}\left(x_{j, k}\right)=f^{\prime}\left(x_{j, k}\right), k \in \mathbb{Z}
\end{gathered}
$$




$$
L_{j} f=f \text { for all } f \in V_{j} .
$$

Theorem 3 Let $f(x) \in L_{2 \pi}^{2}$, and its trigonometric wavelet approximation is $L_{J} f$, then we have

$$
\left\|f(x)-L_{J} f(x)\right\|_{L_{2 \pi}^{2}} \leq C 2^{-2(J+1)}
$$

where $C$ is a positive constant value.

Proof. See [17].

Lemma 2 (The operational matrix of scaling function derivative). (See [20]) The differentiation of vector $\Phi$ in 5 can be expressed as [20]

$$
\Phi^{\prime}=D_{\phi} \Phi
$$

where $D_{\phi}$ is $2^{J+2} \times 2^{J+2}$ operational matrix of derivative for trigonometric scaling function. Suppose

$$
\left(\phi_{j, m}^{s}(x)\right)^{\prime}=\sum_{k=0}^{2^{j+1}-1}\left[a_{k, m}^{s} \varphi_{j, k}^{0}(x)+b_{k, m}^{s} \varphi_{j, k}^{1}(x)\right]
$$

where $m=0,1, \cdots, 2^{j+1}-1$ and $s=0,1$. So the matrix $D_{\phi}$ can be respresented as a block matrix as

$$
D=\left[\begin{array}{cc}
A^{0} & B^{0} \\
A^{1} & B^{1}
\end{array}\right]
$$

where $A^{s}$ and $B^{s}$ are $2^{j+1} \times 2^{j+1}$ matrices. The entries of matrices $A^{s}$ and $B^{s}$ may be find by using

$$
A^{s}=\left(a_{k, m}^{s}\right)=\left(\phi_{j, k}^{s}\right)^{\prime}\left(x_{j, m}\right), \quad B^{s}=\left(b_{k, m}^{s}\right)=\left(\phi_{j, k}^{s}\right)^{\prime \prime}\left(x_{j, m}\right)
$$

where $A^{0}$ is a $2^{j+1} \times 2^{j+1}$ zero matrix, $A^{1}$ is a $2^{j+1} \times 2^{j+1}$ identity matrix. Using $x_{j, m}=\frac{m \pi}{2^{j}}$ we get

$$
\phi_{j, k}^{s}\left(x_{j, m}\right)=\phi_{j, 0}^{s}\left(\frac{(m-k) \pi}{2^{j}}\right)=\phi_{j, 0}^{s}\left(x_{j, m-k}\right)
$$

Using 7 and $B^{s}=\left(b_{k, m}^{s}\right)=\left(\phi_{j, k}^{s}\left(x_{j, m}\right)\right)^{\prime \prime}=\left(\phi_{j, 0}^{s}\left(x_{j, m-k}\right)\right)^{\prime \prime} s=0,1$ we get

$$
B^{0}=\left(b_{k, m}^{0}\right)=\left(\phi_{j, 0}^{0}\right)^{\prime \prime}\left(x_{j, m-k}\right)= \begin{cases}\frac{1}{2} \frac{\cos ((m-k) 2 \pi)}{\sin ^{2}\left(\frac{m-k}{2^{j+1}} \pi\right)} & k \neq m \\ \frac{-1}{2^{2 j+1}} \sum_{p=0}^{2^{j+1}} \sum_{k=1}^{p} \frac{p(p+1)(2 p+1)}{6} & k=m\end{cases}
$$

and

$$
B^{1}=\left(b_{k, m}^{1}\right)=\left(\phi_{j, 0}^{1}\right)^{\prime \prime}\left(x_{j, m-k}\right) \begin{cases}-\cot \left(\frac{m-k}{2^{j+1}} \pi\right) & k \neq m \\ 0 & k=m\end{cases}
$$

for $k, m=0,1, \cdots, 2^{j+1}-1$.

\section{Procedure Solution Using the Trigonometric Scaling Function}

In this section, we first give the computational schemes for Equation (1) with the Newton-Cotes formulas. For either one of these rules, we can make a more accurate approximation by breaking up the interval $[0,1]$ into some number $N$ of subintervals. This is called a composite rule, extended rule, or iterated rule. For example, the composite trapezoidal rule for the discretization form of (1) can be stated as 


$$
\begin{aligned}
& \sum_{i=0}^{2} \mu_{i}(x) u^{(i)}(x)=g(x)+\frac{1}{N}\left(\frac{k(x, 0) u(0)}{2}+\sum_{k=1}^{N-1}\left(k\left(x, \frac{k}{N}\right) u\left(\frac{k}{N}\right)\right)+\frac{k(x, 1) u(1)}{2}\right) \\
& 0 \leq x \leq 1, u(0)=u_{0}, u(1)=u_{1}
\end{aligned}
$$

where the subintervals have the form $[k h,(k+1) h]$, with $h=\frac{1}{N}$ and $k=0,1, \cdots, N-1$. By introducing a basis $\left\{\phi_{j, k}\right\}$ for the subspace $V_{J}$, the coefficients vector $u(x)$ of the discrete solution is defined by

$$
G L_{j} u(x)=\sum_{k=0}^{2^{j+1}-1}\left[a_{k} \varphi_{j, k}^{0}(x)+b_{k} \varphi_{j, k}^{1}(x)\right]=C^{\mathrm{T}} \Phi
$$

where $C$ is $2^{J+2} \times 1$ unknown vector defined similar to (5). By substituting $L_{j} u(x)$ and using Lemma (2) in (1) we have a linear system. Now for determining unknown coefficients vector $C$ or $a_{k}$ and $b_{k}$, we choose collocation method with choosing collocation points as

$$
t_{\tau}=a+\frac{\tau(b-a)}{2^{J+2}}=\frac{\tau \pi}{2^{J+1}} ; \quad \tau=0,1, \cdots, 2^{J+2}-1
$$

Thus we have

$$
\begin{aligned}
& \sum_{i=1}^{2} \mu_{i}\left(t_{\tau}\right) L_{j} u^{(i)}\left(t_{\tau}\right)-\frac{1}{N} \sum_{k=1}^{N-1}\left(k\left(t_{\tau}, \frac{k}{N}\right) L_{j} u\left(\frac{k}{N}\right)\right) \\
& =\frac{1}{2 N}\left(k\left(t_{\tau}, 0\right) L_{j} u_{0}+k\left(t_{\tau}, 1\right) L_{j} u_{1}\right)+g\left(t_{\tau}\right)-\mu_{0}\left(t_{\tau}\right),
\end{aligned}
$$

By using Lemma 2 and after summarizing Equation (13) can be rewritten as the matrix form $Z X=F$ where $k=0,1, \cdots, 2^{J+1}-1, \quad i, j=1, \cdots, 2^{J+1}$, and $\tau=0,1, \cdots, 2^{J+2}-1$. Now, let us calculate the entries $z_{m, s}^{m, s}, m, s=1,2$ and $f\left(t_{\tau}\right)$ in the system matrix.

$$
\begin{aligned}
& Z_{2^{J+2} \times 2^{J+2}}=\left[\begin{array}{ll}
Z_{1,1} & Z_{1,2} \\
Z_{2,1} & Z_{2,2}
\end{array}\right] \\
& Z_{1,1}=\left\{\mu_{1}\right\} B^{0}-\left\{\frac{1}{N} \sum_{k=1}^{N-1}\left(k\left(t_{\tau}, \frac{k}{N}\right) L_{J} u\left(\frac{k}{N}\right)\right)\right\} I=\left(z_{i, j}^{1,1}\right)_{2^{J+1} \times 2^{J+1}} \\
& Z_{2,1}=\left\{\mu_{1}\right\} B^{1}+\left\{\mu_{0}-\frac{1}{N} \sum_{k=1}^{N-1}\left(k\left(t_{\tau}, \frac{k}{N}\right) L_{J} u\left(\frac{k}{N}\right)\right)\right\} I=\left(z_{i, j}^{2,1}\right)_{2^{J+1} \times 2^{J+1}} \\
& Z_{1,2}=B^{0}\left\{\left(\mu_{0}+\mu_{1} B^{1}\right)\right\}-\left\{\frac{1}{N} \sum_{k=1}^{N-1}\left(k\left(t_{\tau}, \frac{k}{N}\right) L_{J} u\left(\frac{k}{N}\right)\right)\right\} I=\left(z_{i, j}^{1,2}\right)_{2^{J+1} \times 2^{J+1}} \\
& Z_{2,2}=\left\{\mu_{0}\right\} B^{1}+\mu_{1}\left(B^{0}+B^{12}\right)-\left\{\frac{1}{N} \sum_{k=1}^{N-1}\left(k\left(t_{\tau}, \frac{k}{N}\right) L_{J} u\left(\frac{k}{N}\right)\right)\right\} I=\left(z_{i, j}^{2,2}\right)_{2^{J+1} \times 2^{J+1}} \\
& X_{2^{J+2} \times 1}=\left[\begin{array}{l}
X_{1} \\
X_{2}
\end{array}\right] \quad X_{1}=\left(a_{k}\right)_{2^{J+1} \times 1} X_{2}=\left(b_{k}\right)_{2^{J+1} \times 1} \\
& F=\left(f\left(t_{\tau}\right)\right)_{2^{j+2} \times 1} \quad f\left(t_{\tau}\right)=\frac{1}{2 N}\left(k\left(t_{\tau}, 0\right) L_{J} u_{0}+k\left(t_{\tau}, 1\right) L_{J} u_{1}\right)+g\left(t_{\tau}\right)-\mu_{0}\left(t_{\tau}\right)
\end{aligned}
$$

where the matrix $A^{0}, A^{1}, B^{0}$, and $B^{1}$ defined in Lemma (2), and $I$ is a $2^{j+1} \times 2^{j+1}$ identity matrix. So the unknown function $u_{J}(x)$ can be found. Note that we find these function by MATLAB.

\section{Numerical Example}

To support our theoretical discussion, we applied the method presented in this paper to several examples. The 
main objective here is to solve these two examples using the trigonometric scaling function and compare our results with exact solution.

Example 4 Consider the second-order the Fredholm Linear Integro-Differential Equation

$$
y^{\prime \prime}(x)+4 x y^{\prime}=-\frac{8 x^{4}}{\left(x^{2}+1\right)^{3}}-2 \int_{0}^{1} \frac{t^{2}+1}{\left(x^{2}+1\right)^{2}} y(t) \mathrm{d} t
$$

with the mixed conditions $y(0)=1$ and $y(1)=\frac{1}{2}$. The exact solution of this problem is $\left(x^{2}+1\right)^{(-1)}$. We apply the suggested method with $J=1$ and $J=2$. The behavior of the approximate solution using the proposed method with $J=1, \quad J=2$ and the exact solution are presented in Figure 1. In Table 1, we give the errors $E_{J}$ of matrix $A$ for different values of $J$. From this figure, it is clear that the proposed method can be considered as an efficient method to solve the linear integral equations. From Table 1 we see the errors decrease rapidly as $J$ increase.

In Table 2 we compare the new method with $J=1, \quad J=2$ and $J=3$ together with the exact solution. For the purpose of comparison we defined the meximum error for $u_{J}(x)$ as

$$
E_{J}=\left\|u_{J}(x)-u(x)\right\|_{\infty}=\max \left\{\left|u_{J}(x)-u(x)\right|, a \leq x \leq b\right\}
$$

Example 5 Consider the following second-order the Fredholm Linear Integro-Differential Equation

$$
x^{2} u^{\prime \prime}(x)+50 x u^{\prime}=\frac{1-\mathrm{e}^{(x+1)}}{x+1}\left(x^{2}+50 x-35\right) \mathrm{e}^{x}+\int_{0}^{1} \mathrm{e}^{x t} u(t) \mathrm{d} t
$$

with the initial conditions $u(0)=1, u(1)=\mathrm{e}$ and exact solution $\mathrm{e}^{x}$. This problem is solved by the same methods applied in example (4). Results are shown in Figure 2. From this figure, it is clear that the proposed method can be considered as an efficient method to solve the linear integral equations. For the purpose of comparison in Table 3 we give the errors $E_{J}$ of matrix $A$ for different values of $J$. From Table 4 we see the errors decrease rapidly as $J$ increase. In Table 4 we compare the new method with $J=1, J=2$ and $J=3$ together with the exact solution.

Table 1. The maximum error matrix $A$ from Example 4.

\begin{tabular}{|c|c|c|c|c|}
\hline$t_{i}$ & Exact solution & $u_{j}$ for $J=1$ & $u_{j}$ for $J=2$ & $u_{j}$ for $J=3$ \\
\hline 0 & $1.00000000 e-00$ & $1.00000000 e-00$ & $1.00000000 e-00$ & $1.00000000 e-00$ \\
\hline 0.125 & $9.84643786 e-01$ & $9.81250761 e-01$ & $9.84057813 e-01$ & $9.84643237 e-01$ \\
\hline 0.2500 & $9.41218794 e-01$ & $9.4075171 e-01$ & $9.41102894 e-01$ & $9.41218075 e-01$ \\
\hline 0.3750 & $8.76735484 e-01$ & $8.76170942 e-01$ & $8.76731207 e-01$ & $8.76730782 e-01$ \\
\hline 0.5000 & $8.00001246 e-01$ & $8.00180793 e-01$ & $8.00080476 e-01$ & $8.00001107 e-01$ \\
\hline 0.6250 & $7.19101507 e-01$ & $7.18017064 e-01$ & $7.19852079 e-01$ & $7.19101672 e-01$ \\
\hline 0.7500 & $6.40018961 e-01$ & $6.31094827 e-01$ & $6.4180973 e-01$ & $6.40018843 e-01$ \\
\hline 0.8750 & $5.66434082 e-01$ & $5.50789164 e-01$ & $5.66027815 e-01$ & $5.66434729 e-01$ \\
\hline
\end{tabular}

\begin{tabular}{cccccc}
\hline$J$ & $J=1$ & $J=2$ & $J=3$ & $J=4$ & $J=5$ \\
\hline$E_{J}$ & $2.5 \times 10^{-3}$ & $3.5 \times 10^{-5}$ & $5.2 \times 10^{-6}$ & $2.6 \times 10^{-7}$ & $5.8 \times 10^{-8}$ \\
\hline
\end{tabular}

Table 2. Error analysis and numerical results of Example 4.

Table 3. The maximum error matrix A from Example 5.

\begin{tabular}{ccccc}
\hline$J$ & $J=1$ & $J=2$ & $J=3$ & $J=4$ \\
\hline$E_{J}$ & $2.9 \times 10^{-5}$ & $5.2 \times 10^{-6}$ & $5.9 \times 10^{-7}$ & $2.2 \times 10^{-7}$ \\
\hline
\end{tabular}



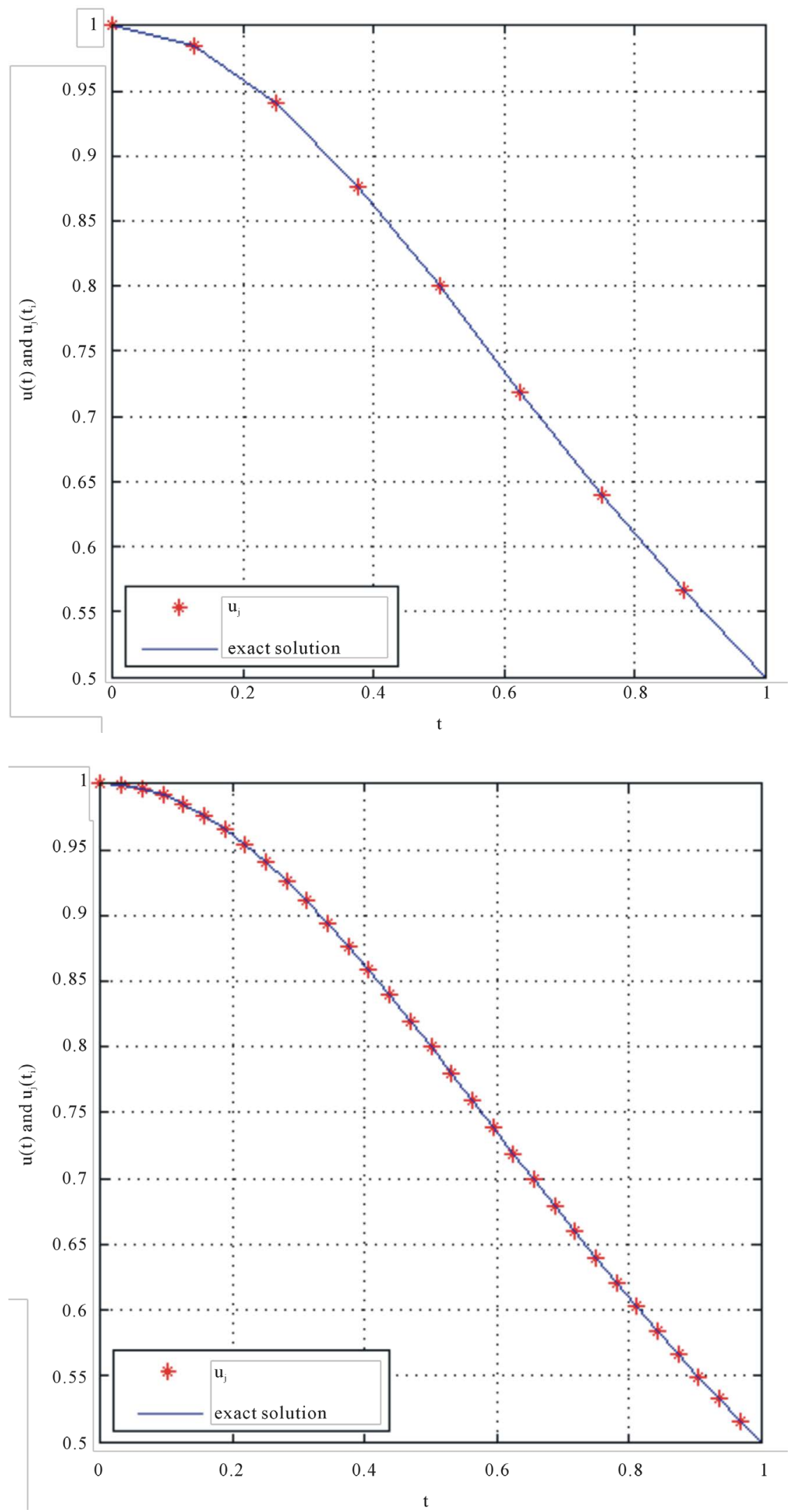

Figure 1. Result EX.4 for $J=1$ and $N=7$; Result EX. 4 for $J=2$ and $N=7$. 

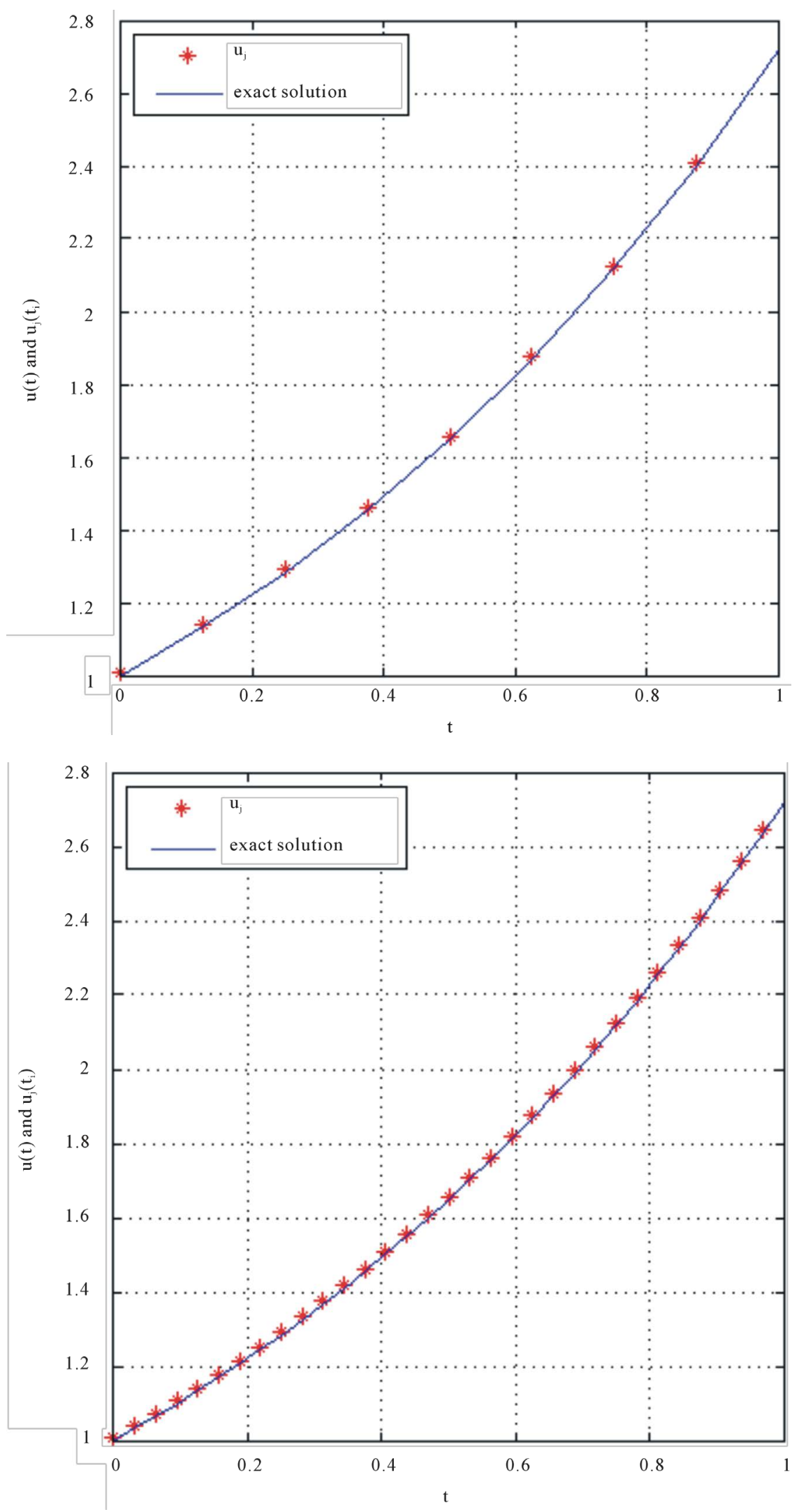

Figure 2. Result EX.5 for $J=1$ and $N=7$; Result EX.5 for $J=2$ and $N=7$. 
Table 4. Error analysis and numerical results of Example 5.

\begin{tabular}{ccccc}
\hline$t_{i}$ & Exact solution & $u_{j}$ for $J=1$ & $u_{j}$ for $J=2$ & $u_{j}$ for $J=3$ \\
\hline 0 & 1.00000000 & 1.00104921 & 1.00002430 & 1.00000013 \\
0.125 & 1.13310019 & 1.13048310 & 1.13307891 & 1.13310007 \\
0.2500 & 1.28400186 & 1.28178029 & 1.28314720 & 1.28400134 \\
0.3750 & 1.45500374 & 1.45304829 & 1.45456781 & 1.45500352 \\
0.5000 & 1.64870391 & 1.64910273 & 1.64820756 & 1.64870372 \\
0.6250 & 1.86820582 & 1.86705912 & 1.86835405 & 1.868820565 \\
0.7500 & 2.11704076 & 2.11078316 & 2.11527804 & 2.11704052 \\
0.8750 & 2.39893108 & 2.39719802 & 2.39884240 & 2.39893214 \\
\hline
\end{tabular}

\section{Conclusion}

Our results indicate that the method with the trigonometric scaling bases can be regarded as a structurally simple algorithm that is conventionally applicable to the numerical solution of IDEs. In addition, although we have restricted our attention to linear Fredholm IDEs, we expect the method to be easily extended to more general IDEs. the presented method which is based on the trigonometric scaling function is proposed to find the approximate solution. A comparison of the exact solution reveals that the presented method is very effective and convenient. Nevertheless, as Figure 1 and Figure 2 illustrate, the error of the trigonometric scaling bases shows that the accuracy improves with increasing $J$, hence for better results, using number $J$ is recommended. Also form the obtained approximate solution, we can conclude that the proposed method gives the solution in an excellent agreement with the exact solution. All computations are done using MATLAB programming.

\section{Acknowledgements}

The authors are very grateful to the editor for carefully reading the paper and for their comments and suggestions which have improved the paper.

\section{References}

[1] Desmond, R.A., Weiss, H.L., Arani, R.B., Soong, S.-J., Wood, M.J., Fiddian, P., Gnann, J. and Whitley, R.J. (2002) Clinical Applications for Change-Point Analysis of Herpes Zoster Pain. Journal of Pain and Symptom Management, 23, 510-516. http://dx.doi.org/10.1016/S0885-3924(02)00393-7

[2] Wazwaz, D.D., Dimitrova, M.B. and Dishliev. A.B. (2000) Oscillation of the Bounded Solutions of Impulsive Differential-Difference Equations of Second Order. Applied Mathematics and Computation, 114, 61-68. http://dx.doi.org/10.1016/S0096-3003(99)00102-2

[3] Gulsu, M. and Sezer, M. (2006) A Taylor Polynomial Approach for Solving Differential-Difference Equations. Journal of Computational and Applied Mathematics, 186, 349-369. http://dx.doi.org/10.1016/j.cam.2005.02.009

[4] Maleknejad, K., Mirzaee, F. and Abbasbandy, S. (2004) Solving Linear Integro-Differential Equations System by Using Rationalized Haar Functions Method. Applied Mathematics and Computation, 155, 317-328. http://dx.doi.org/10.1016/S0096-3003(03)00778-1

[5] Maleknejad, K., Tavassoli, M. and Kajani, M. (2004) Solving Linear Integro-Differential Equation by Galerkin Methods with Hybrid Functions. Journal of Computational and Applied Mathematics, 159, 603-612. http://dx.doi.org/10.1016/j.amc.2003.10.046

[6] Ganesh, M. and Sloan, I.H. (1999) Optimal Order Spline Methods for Nonlinear Differential and Integro-Differential Equations. Applied Numerical Mathematics, 29, 445-478. http://dx.doi.org/10.1016/S0168-9274(98)00067-1

[7] Dascoglua, A. and Sezer, M. (2005) Chebyshev Polynomial Solutions of Systems of Higher-Order Linear FredholmVolterra Integro-Differential Equations. Journal of the Franklin Institute, 342, 688-701. http://dx.doi.org/10.1016/j.jfranklin.2005.04.001

[8] Faour, A.L. and Saeed, R.K. (2006) Solution of a System of Linear Volterra Integral and Integro-Differential Equations by Spectral Method. Journal of Al-Nahrain University/Science, 62, 30-46. 
[9] Danfu, H. and Xufeng, A.S. (2007) Numerical Solution of Integro-Differential Equations by Appling CAS Wavelet Operational Matrix of Integration. Applied Mathematics and Computation, 194, 460-466. http://dx.doi.org/10.1016/j.amc.2007.04.048

[10] Baker, C. and Tang, A. (1997) Stability of Continuous Implicit Runge-Kutta Methods for Volterra Integro-Differential Systems with Unbounded Delays. Applied Numerical Mathematics, 24, 153-173. http://dx.doi.org/10.1016/S0168-9274(97)00018-4

[11] El-Sayed, S., Kaya, D. and Zarea, S. (2004) The Decomposition Method Applied to Solve High Order Linear VolterraFredholm Integro-Differential Equations. International Journal of Nonlinear Sciences and Numerical Simulation, 52, 105-112.

[12] Sezer, M. and Gulsu, M. (2007) Polynomial Solution of the Most General Linear Fredholm-Volterra Integro DifferentialDifference Equations by Means of Taylor Collocation Method. Applied Mathematics and Computation, 185, 646-657. http://dx.doi.org/10.1016/j.amc.2006.07.051

[13] Sezer, M. and Gulsu, M. (2005) A New Polynomial Approach for Solving Difference and Fredholm Integro-Difference Equations with Mixed Argument. Applied Mathematics and Computation, 171, 332-344. http://dx.doi.org/10.1016/j.amc.2005.01.051

[14] Chui, C.K. and Mhaskar, H.N. (1993) On Trigonometric Wavelets. Constructive Approximation, 9, 167-190. http://dx.doi.org/10.1007/BF01198002

[15] Prestin, J. (2001) Trigonometric Wavelets. In: Jain, P.K., et al., Eds., Wavelet and Allied Topics, Narosa Publishing House, New Delhi, 183-217.

[16] Themistoclakis, W. (1999) Trigonometric Wavelet Interpolation in Besov Spaces. Facta Univ. (Nis) Ser. Math. Inform, 14, 49-70.

[17] Quak, E. (1996) Trigonometric Wavelets for Hermite Interpolation. Department of Mathematics, Texas A. M. University, 65, 683-722.

[18] Chen, W.S. and Lin, W. (1997) Hadamard Singular Integral Equations and Its Hermite Wavelet Methods. Proceedings of the 5th International Colloquium on Finite Dimensional Complex Analysis, Beijing, 13-22.

[19] Chen, W.S. and Lin, W. (2002) Trigonometric Hermite Wavelet and Natural Integral Equations for Stockes Problem. International Conference on Wavelet Analysis and Its Applications, Guangzhou, 73-86.

[20] Lakestani, M. and Saray, B.N. (2010) Numerical Solution of Telegraph Equation Using Interpolating Scaling Functions. Computer and Mathematics with Application, 60, 1964-1972. http://dx.doi.org/10.1016/j.camwa.2010.07.030 